\title{
Photodynamic therapy for mycosis fungoides: a case series and review of the literature
}

\author{
Robert E. Hunger, Mohamed Sallam, Nikhil Yawalkar
}

Department of Dermatology, Inselspital, University of Bern

Freiburgstrasse 10, CH-3011 Bern, Switzerland

\begin{abstract}
Mycosis fungoides (MF) is the most common form of cutaneous T cell lymphoma. In early stages of the disease, topical therapeutic approaches like steroids, chemotherapy, phototherapy or spot radiation therapy are most commonly used. Photodynamic therapy (PDT) is widely executed in the treatment of actinic keratosis and superficial basal cell carcinoma. The effective use of PDT for early forms of MF has been previously demonstrated in a series of cases. In this instance, the treatment of MF ( $n=6,11$ lesions) with methyl alanine PDT (MAL-PDT) in $73 \%$ of the treated lesions showed a complete response. Within the timeframe of 25-51 months, no recurrence of the successfully treated lesions was observed, on the contrary some of the patients developed new lesions on different sites. Hence, this case study shows that patients having a single or few MF lesions can be successfully treated by PDT.
\end{abstract}

Key words: photodynamic therapy, 5-aminolevulinic acid, methyl aminolevulinate.

Corresponding author: robert.hunger@insel.ch. Vestnik Dermatologii i Venerologii 2015; 1: 24-27.

\section{Introduction}

Cutaneous T cell lymphoma (CTCL) is a heterogeneous group of malignant $T$ lymphocytes arising primarily in the skin [1]. The most common form is mycosis fungoides (MF). The clinical presentation is highly variable. While the early form is characterized by localized skin lesions such as red patches and plaques, late stages can be identified by generalized patches, plaques, tumors and erythroderma [2]. Patients with early forms of MF, i.e. localized patches and plaques covering less than $10 \%$ of the body surface, have an excellent prognosis with the potential for a longer life-span. However, patients with generalized form have a high risk of disease progression leading to death [3]. Until now there are no curative options and all therapies aim to relieve the patients from the symptoms that accompany either form of MF. The current treatment modalities for localized forms are highly potent topical steroids, chemotherapy, phototherapy and radiation therapy [4].

Photodynamic therapy (PDT) is widely used for the treatment of actinic keratosis and superficial basal cell carcinoma where its efficacy and safety has been well documented [5]. The most frequently used is 5-aminolaevulinic acid (ALA) or methyl-aminolaevulinic acid (MAL) which is applied to the skin tumor in a cream under occlusion. During the incubation time of 2-4 hours, phototoxic porphyrins preferentially accumulate in the neoplastic tissue. Death of the neoplastic cells is then induced by illumination with an appropriate light source. However, red light is preferred over blue light as it penetrates deeper into the lesion. The advantages of PDT include excellent cosmetic results and the lack of systemic toxicity. The major disadvantage is that it may induce considerable pain especially during illumination [6] which in turn make concomitant topical or systemic interventions such as oral anaesthetic agents, nerve blocks or topical cooling necessary [7].

\section{Patients and Methods \\ Patients}

Six patients which comprises of 1 woman and 5 men whose ages range from 32-81 and with a mean age 56.5 years were included in the analysis (Table 1). Prior to the study, each patient were histologically confirmed with MF. A total of 11 lesions were treated. Given that all lesions were treated repeatedly, 10 were treated 2-4 times while one lesion was treated 14 times. All patients were regularly followed at our outpatient clinic. The median follow-up time of the patients with a complete response was 36.1 months (range 25-51).

Therapeutic procedure and assessment of effectiveness

A thick layer of $16.8 \%$ MAL cream (Metvix, Galderma, Switzerland) was applied topically to the lesion. The lesion was covered with an occlusive dressing to be protected from any light source and incubated for a 3 hour-period. Thereafter, the dressing was removed and the MAL cream was washed off with saline. The lesions were then illuminated with red light from a light-emitting diode (LED, Actilite ${ }^{\circledR}$, Galderma, Switzerland) at a mean wavelength of $630 \mathrm{~nm}$ with a total dose of $37.5 \mathrm{~J} / \mathrm{cm}^{2}$. Cold water spray was ap- 
plied during illumination to reduce pain. Furthermore, $1 \mathrm{~g}$ paracetamol/acetaminophen (Dafalgan $®$ ) was given orally 1 hour prior to illumination for the reduction and relief of pain. As indicated in Table, several sessions were made for each patient. The therapeutic effectiveness was also clinically assessed. The response was graded as complete response (95-100\%), partial response (50-95\%), no response $(<50 \%)$, or progressive disease. Moreover, photographs were taken with a digital camera under controlled lighting conditions before and after the treatment sessions.

\section{Results}

As shown in Table 1, 8 of the 11 (73\%) treated lesions showed a complete response, 2 had a partial response and 1 lesion showed no response. Representative examples of successfully treated lesions are shown in Figure 1. All lesions were treated repeatedly, most commonly 2-4 times. However, only one lesion which was located on the penis (patient 6) was treated 14 times. As this lesion did not show a satisfactory result, it was further treated more extensively with spot radiation therapy. During the course of treatment, one patient (patient 3) developed a contact allergic reaction to Metvix $®$ (confirmed by patch testing) after the last (4th) therapeutic session. No other side effects except pain and the local reaction were detected. During the whole follow-up period (mean 36.1 month, range 25-51) none of the patients with a complete response showed a relapse of the successfully treated
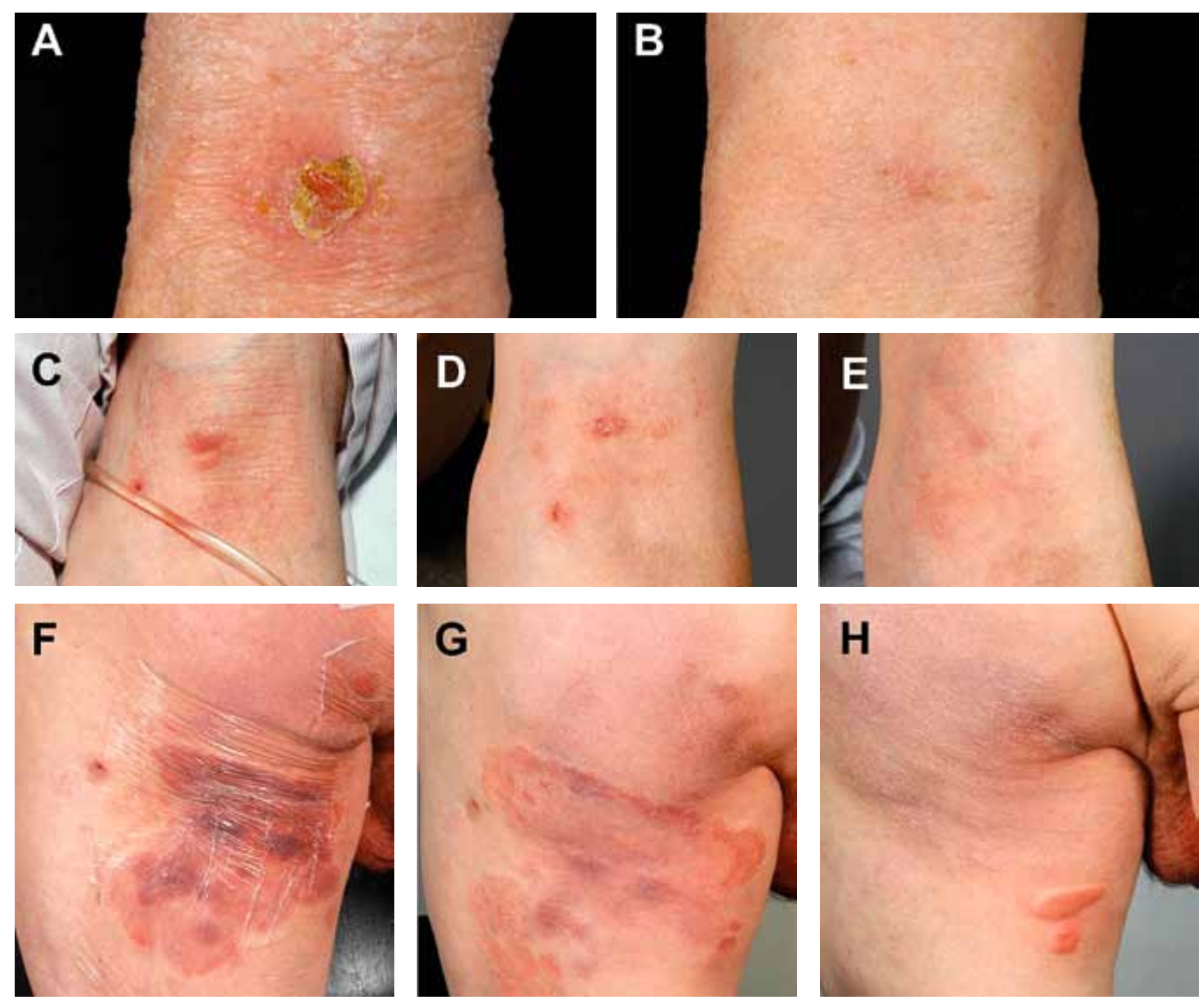

Representative examples of successfully treated patients. Ulcerating tumor on the left forearm of patient 1 before

Figure. (A) and 3 month after last treatment (B). Tumor on the left arm of patient 4 before (C) 2 weeks (D) and 4 month (E) after last treatment. Plaque on the left thigh of patient 4 before (F) 3 weeks (G) and 12 month $(\mathrm{H})$ after last treatment 
Tab Patients characteristics and clinical response

\begin{tabular}{|c|c|c|c|c|c|c|c|c|c|}
\hline $\begin{array}{l}\text { patient } \\
\text { number }\end{array}$ & sex & age & $\begin{array}{c}\text { size of } \\
\text { lesions } \\
{[\mathrm{mm}]}\end{array}$ & localization & $\begin{array}{c}\text { characteristics of } \\
\text { lesion }\end{array}$ & $\begin{array}{l}\text { number of } \\
\text { treatments }\end{array}$ & response & $\begin{array}{c}\text { re- } \\
\text { cur- } \\
\text { rence }\end{array}$ & $\begin{array}{l}\text { follow } \\
\text { up time } \\
\text { [month] }\end{array}$ \\
\hline 1 & $f$ & 81 & 25 & forearm & tumor & 3 & $\mathrm{CR}$ & no & 25 \\
\hline 2 & $\mathrm{~m}$ & 62 & 45 & left thigh & ulcerating tumor & 2 & $\mathrm{CR}$ & no & 24 \\
\hline \multirow[t]{2}{*}{3} & $\mathrm{~m}$ & 32 & 110 & buttocks & patch & 4 & NR & n.a. & n.a. \\
\hline & & & 18 & lower back & plaque & 4 & $\mathrm{CR}$ & no & 26 \\
\hline \multirow[t]{3}{*}{4} & $\mathrm{~m}$ & 72 & 20 & buttocks left side & tumor & 2 & $\mathrm{CR}$ & no & 38 \\
\hline & & & 95 & left thigh & plaque & 2 & CR & no & 51 \\
\hline & & & 15 & left crook of the arm & tumor & 2 & CR & no & 51 \\
\hline \multirow[t]{3}{*}{5} & $\mathrm{~m}$ & 42 & 85 & right upper arm & plaque & 3 & PR & n.a. & n.a. \\
\hline & & & 75 & right neck & patch & 2 & CR & no & 37 \\
\hline & & & 95 & left neck & patch & 2 & CR & no & 37 \\
\hline 6 & $\mathrm{~m}$ & 50 & 18 & penis & ulcerative plaque & 14 & PR & n.a. & n.a. \\
\hline
\end{tabular}

lesions. Interestingly, 2 patients presented lesions with a complete response and some lesions only with partial response. After successful treatment, one patient (patient 4) developed a new lesion adjacent to the treatment site after one year (Figure $1 \mathrm{H}$ ), while the previously treated lesions remained without any sign of recurrence.

\section{Discussion}

In our study population, $73 \%$ of all treated MF lesions showed a complete clinical response (CR). This is comparable with data presented in previous case series with MF lesions. Wolf et al. [8] reported 1994 a complete clinical response (CR) in 3 out of 3 treated lesions. In $2001 \mathrm{Ed}$ strom et al. [9] reported a CR in 7 out of 12 treated lesions and in 2008 he [10] summarized long term follow up data demonstrating that the treated lesions remained free of recurrence although most patients developed progressive disease at other locations. Zane et al [11] reported a CR in 4 out of 5 treated lesions and Kim et al [12] in 5 of 10 lesions. The only prospective controlled study performed so far, reported a CR in 11 of 21 treated lesions and an overall clinical response rate of $75 \%$ [13]. Most case series used MAP-PDT, however, ALA-PDT also seem to be efficient. During the whole observation period, no recurrence of the successfully treated lesions was observed. However, one patient developed two fresh lesions in close proximity to the previously treated lesions. Similar findings have also been reported by previous studies $[10,13]$.

Currently, the recommendation for the treatment of the early stage of MF, if only one or few lesions are present are local radiotherapy, topical phototherapy, topical chemotherapy or highly potent topical steroids [3]. The use of PDT has several advantages compared to these treatment modalities as follows: 1. In comparison to topical chemotherapy PDT has no systemic toxicity, 2. Unlike phototherapy or radiotherapy PDT also has no carcinogenic effects, 3. PDT usually only requires few sessions, no repeated applications over weeks as for topical phototherapy or topical steroid is necessary, 4 . The cosmetic results are excellent, 5 . The whole procedure of PDT is done by the health care personnel and it is also suited for patients with dissatisfactory compliance. The disadvantage of PDT is that illumination often causes a painful sensation and it is relatively expensive. Nevertheless, the common side effect which is pain may be alleviated with the newly introduced procedure of daylight-PDT [14, 15]. However, no data for the efficacy of daylight-PDT in patients with MF is so far available.

In conclusion, MAL-PDT is an efficient therapeutic option for unilesional or paucilesional forms of MF. The recurrence rate seems to be low. Correspondingly, a controlled longterm follow up studies are required to validate these data. I 


\section{Literature}

1. Willemze R., Jaffe E.S., Burg G., Cerroni L., Berti E., Swerdlow S.H. et al. WHO-EORTC classification for cutaneous lymphomas. Blood. 2005;105(10):3768-85. Epub 2005/02/05.

2. Jawed S.I., Myskowski P.L., Horwitz S., Moskowitz A., Querfeld C. Primary cutaneous T-cell lymphoma (mycosis fungoides and Sezary syndrome): part I. Diagnosis: clinical and histopathologic features and new molecular and biologic markers. Journal of the American Academy of Dermatology. 2014; 70(2): 205 e1-16; quiz 21-2. Epub 2014/01/21.

3. Jawed S.I., Myskowski P.L., Horwitz S., Moskowitz A., Querfeld C. Primary cutaneous T-cell lymphoma (mycosis fungoides and Sezary syndrome): part II. Prognosis, management, and future directions. Journal of the American Academy of Dermatology. 2014; 70(2): 223 e1-17; quiz 40-2. Epub 2014/01/21.

4. Willemze R., Dreyling M. Primary cutaneous lymphomas: ESMO Clinical Practice Guidelines for diagnosis, treatment and follow-up. Annals of oncology : official journal of the European Society for Medical Oncology / ESMO. 2010;21 Suppl 5:v177-80. Epub 2010/06/29.

5. Braathen L.R., Szeimies R.M., Basset-Seguin N., Bissonnette R., Foley P., Pariser D. et al. Guidelines on the use of photodynamic therapy for nonmelanoma skin cancer: an international consensus. International Society for Photodynamic Therapy in Dermatology, 2005. Journal of the American Academy of Dermatology. 2007:56(1):125-43. Epub 2006/12/28.
6. Wennberg A.M. Pain, pain relief and other practical issues in photodynamic therapy. The Australasian journal of dermatology. 2005; 46 Suppl 3:S3-4; discussion S23-5. Epub 2005/04/30.

7. Warren C.B., Karai L.J., Vidimos A., Maytin E.V. Pain associated with aminolevulinic acid-photodynamic therapy of skin disease. Journal of the American Academy of Dermatology. 2009;61(6):1033-43. Epub 2009/11/21.

8. Wolf P., Fink-Puches R., Cerroni L., Kerl H. Photodynamic therapy for mycosis fungoides after topical photosensitization with 5-aminolevulinic acid. Journal of the American Academy of Dermatology. 1994;31(4):678-80. Epub 1994/10/01.

9. Edstrom D.W., Porwit A., Ros A.M. Photodynamic therapy with topical 5 -aminolevulinic acid for mycosis fungoides: clinical and histological response. Acta dermato-venereologica. 2001; 81(3): 184-8. Epub 2001/09/18.

10. Edstrom D.W., Hedblad M.A. Long-term followup of photodynamic therapy for mycosis fungoides. Acta dermato-venereologica. 2008; 88(3): 288-90. Epub 2008/05/16.

11. Zane C., Venturini M., Sala R., Calzavara-Pinton P. Photodynamic therapy with methylaminolevulinate as a valuable treatment option for unilesional cutaneous T-cell lymphoma. Photodermatology, photoimmunology \& photomedicine. 2006; 22(5): 254-8. Epub 2006/09/05.
12. Kim S.T., Kang D.Y., Kang J.S., Baek J.W., Jeon Y.S., Suh K.S. Photodynamic therapy with methyl-aminolaevulinic acid for mycosis fungoides. Acta dermato-venereologica. 2012; 92(3): 264-8. Epub 2011/12/16.

13. Quereux G., Brocard A., Saint-Jean M., Peuvrel L., Knol A.C., Allix R. et al. Photodynamic therapy with methyl-aminolevulinic acid for paucilesional mycosis fungoides: a prospective open study and review of the literature. Journal of the American Academy of Dermatology. 2013; 69(6): 890-7. Epub 2013/09/18.

14. Rubel D.M., Spelman L., Murrell D.F., See J.A., Hewitt D., Foley P. et al. Daylight PDT with methyl aminolevulinate cream as a convenient, similarly effective, nearly painless alternative to conventional PDT in actinic keratosis treatment: a randomised controlled trial. The British journal of dermatology. 2014. Epub 2014/05/28.

15. Wiegell S.R., Wulf H.C., Szeimies R.M., BassetSeguin N., Bissonnette R., Gerritsen M.J. et al. Daylight photodynamic therapy for actinic keratosis: an international consensus: International Society for Photodynamic Therapy in Dermatology. Journal of the European Academy of Dermatology and Venereology: JEADV. 2012; 26(6): 673-9. Epub 2012/01/04. 
$\Phi$

отодинамическая терапия грибовидного микоза: исследование серии случаев и обзор литературы

Robert E. Hunger, Mohamed Sallam, Nikhil Yawalkar

Отделение дерматологии, клиника Инзельшпиталь, Бернский университет

Берн, Швейцария, Фрайбургштрассе, 10, СН-3011

Грибовидный микоз (ГМ) является наиболее распространенной фрормой Т-клеточной лимсромы кожи. На ранних стадиях заболевания чаще всего используется местная терапия, например стероиды, химиотерапия, фототерапия или точечная лучевая терапия. Фотодинамическая терапия (ФДТ) находит широкое применение при лечении актинического кератоза и поверхностной базальноклеточной карциномы. Эфреективность использования ФДТ для ранних форм ГМ была ранее показана в ряде случаев. В данном случае лечение ГМ ( $n=6,11$ очагов) при помощи ФДТ с метилаланином (МАЛ-ФДТ) обеспечило полную ответную реакцию для 73\% обработанных очагов. На протяжении периода продолжительностью 25-51 мес. не было никаких рецидивов в связи с успешно вылеченными очагами, однако у некоторых пациентов появились новые очаги на различных участках. По результатам данного клинического наблюдения было показано, что пациентов с одним очагом ГМ или небольшим числом очагов ГМ можно успешно лечить при помощи ФДТ.

Ключевые слова: фотодинамическая терапия, 5-аминолавулиновая кислота.

Контактная информация: robert.hunger@insel.ch. Вестник дерматологии и венерологии 2015; (1): 28—31.

Т-клеточная лимфома кожи (ТКЛК) представляет собой разнородную группу злокачественных Т-лимфоцитов, локализующихся преимущественно в коже [1]. Наиболее распространенной фрормой является грибовидный микоз (ГМ). Его клиническая картина в значительной степени изменчива. Ранняя форма характеризуется локализованными кожными очагами, включая красные пятна и бляшки, тогда как поздние стадии можно определить по распространенным пятнам, бляшкам, опухолям и эритродермии [2]. Прогноз для пациентов с ранними формами МФ, т.е. локализованными пятнами и бляшками, занимающими менее $10 \%$ площади поверхности тела, является благоприятным, а ожидаемая продолжительность жизни у них высокая. Вместе с тем, пациенты с распространенными формами характеризуются высоким риском прогрессирования заболевания с последующим летальным исходом [3]. К настоящему моменту времени отсутствуют какие-либо методы излечения данного заболевания, а все виды терапии направлены на облегчение у пациентов симптомов, возникающих при любой фрорме ГМ. В настоящее время к вариантам лечения локализованных форм относятся сильнодействующие топические стероиды, химиотерапия, фототерапия или точечная лучевая терапия [4].
Фотодинамическая терапия (ФДТ) находит широкое применение при лечении актинического кератоза и поверхностной базальноклеточной карциномы, а ее эффрективность и безопасность подтверждены достаточными документальными свидетельствами [5]. Чаще всего используются 5-аминолавулиновая кислота (АЛК) или метиламинолавулиновая кислота (МАЛ), которые наносят на кожные опухоли в лекарственной форме крема под повязкой. В течение инкубационного периода (2-4 4) фрототоксические порфирины накапливаются преимущественно в опухолевых тканях. Последующее освещение соответствующим источником света приводит к гибели опухолевых клеток. Тем не менее красный свет более предпочтителен по сравнению с синим, поскольку он глубже проникает в очаг поражения. К преимуществам ФДТ относятся превосходные косметические результаты и отсутствие системной токсичности. Основным недостатком терапии является тот фракт, что ФДТ может вызывать значительные болевые ощущения, особенно во время облучения [6], что, в свою очередь, обусловливает необходимость применения сопутствующих мер местного или системного действия, например пероральных анестетиков, блокады нервов или местного охлаждения [7]. 


\section{Пациенты и методы}

\section{Пациенты}

В исследование было включено 6 пациентов 1 женщина и 5 мужчин в возрасте от 32 лет до 81 года (средний возраст 56,5 года) (таблица). Диагноз ГМ всем больным был подтвержден гистологически. В общей сложности было обработано 11 очагов, 10 очагов были обработаны 2-4 раза, а один очаг - 14 раз. Все пациенты проходили регулярное последующее наблюдение в нашей амбулаторной клинике. Средний срок последующего наблюдения за пациентами с полной ответной реакцией составил 36,1 мес. (в диапазоне от 25 до 51 мес.).

\section{Терапевтическая процедура и оценка эффрективности}

На очаг наносили толстый слой крема 16,8\% МАЛ (Metvix, Galderma, Швейцария). Очаг покрывали окклюзионной повязкой для защиты от источников света на 3 ч. Затем повязку снимали, а крем МАЛ смывали при помощи фризиологического раствора. После этого очаги облучали красным светом при помощи светоизлучающего диода (СИД, Actilite ${ }^{\circledR}$, Galderma, Швейцария) со средней длиной волны 630 нм, общая доза - 37,5 Дж/см². Во время облучения для смягчения болевых ощущений использовали распылитель с холодной водой. Кроме того, за один час до облучения пациенты получали 1 г парацетамола/ацетаминофрена (Dafalgan $\left.{ }^{\circledR}\right)$ перорально для смягчения и устранения боли. Как указано в таблице, каждый пациент прошел несколько сеансов лечения. Также была проведена клиническая оценка эффрективности терапии. Ответную реакцию оценивали следующим образом: полная ответная реакция (95-100\%), частичная ответная реакция (50-95\%), отсутствие ответной реак- ции (<50\%) или дальнейшее прогрессирование заболевания. Кроме того, при помощи цифровой камеры были сделаны фотографии с контролируемыми условиями освещения до и после сеансов лечения.

\section{Результаты}

Как видно из таблицы, 8 из 11 обработанных очагов (73\%) проявили полную ответную реакцию, 2 очага - частичную ответную реакцию, а 1 очаг не проявил ответной реакции. Наглядные примеры успешно вылеченных очагов приведены на рисунке. Все очаги обрабатывали неоднократно - чаще всего 2-4 раза. Тем не менее только один очаг, расположенный на пенисе (у пациента 6), был обработан 14 раз. Поскольку лечение этого очага не принесло удовлетворительного результата, далее его подвергли точечной лучевой терапии. Во время курса лечения у одного пациента (пациент 3) развилась контактная аллергическая реакция на Metvix ${ }^{\circledR}$ (подтвержденная кожной пробой) после последнего (четвертого) сеанса лечения. Других побочных эффректов, кроме боли и местной реакции, отмечено не было. Во время всего периода последующего наблюдения (в среднем 36,1 мес. в диапазоне от 25 до 51 мес.) ни у одного из пациентов с полной ответной реакцией не было выявлено рецидивов в отношении успешно излеченных очагов. Примечательно, что у двоих пациентов были отмечены очаги с полной ответной реакцией и ряд очагов с частичной ответной реакцией. После успешного лечения у одного пациента (пациент 4) появился новый очаг рядом с обработанным участком по прошествии одного года (см. рисунок Н), в то время как ранее обработанные очаги по-прежнему не проявляли признаков рецидива.

Таблица Характеристика пациентов и клиническая эффрективность терапии

\begin{tabular}{|c|c|c|c|c|c|c|c|c|c|}
\hline $\begin{array}{l}\text { № па- } \\
\text { циен- } \\
\text { та }\end{array}$ & Пол & $\begin{array}{l}\text { В03- } \\
\text { раст }\end{array}$ & $\begin{array}{c}\text { Размер } \\
\text { очага } \\
\text { [мм] }\end{array}$ & Локализация & $\begin{array}{c}\text { Характеристика } \\
\text { поражения }\end{array}$ & $\begin{array}{c}\text { Коли- } \\
\text { чество } \\
\text { процедур }\end{array}$ & $\begin{array}{c}\text { Ответ } \\
\text { на терапию }\end{array}$ & Рецидив & $\begin{array}{c}\text { Время } \\
\text { наблюдения } \\
\text { [месяц] }\end{array}$ \\
\hline 1 & ж & 81 & 25 & Предплечье & Опухоль & 3 & Полный ответ & Отсутствие & 25 \\
\hline 2 & M & 62 & 45 & Левое бедро & Изьязвленная опухоль & 2 & Полный ответ & Отсутствие & 24 \\
\hline \multirow[t]{2}{*}{3} & M & 32 & 110 & Ягодицы & Пятно & 4 & Отсутствие ответа & Нет данных & Нет данных \\
\hline & & & 18 & Поясничная область & Бляшка & 4 & Полный ответ & Отсутствие & 26 \\
\hline \multirow[t]{3}{*}{4} & M & 72 & 20 & Левая ягодица & Опухоль & 2 & Полный ответ & Отсутствие & 38 \\
\hline & & & 95 & Левое бедро & Бляшка & 2 & Полный ответ & Отсутствие & 51 \\
\hline & & & 15 & Левый локтевой сгиб & Опухоль & 2 & Полный ответ & Отсутствие & 51 \\
\hline \multirow[t]{3}{*}{5} & M & 42 & 85 & Правое плечо & Бляшка & 3 & Частичный ответ & Нет данных & Нет данных \\
\hline & & & 75 & Правая половина шеи & Пятно & 2 & Полный ответ & Отсутствие & 37 \\
\hline & & & 95 & Левая половина шеи & Пятно & 2 & Полный ответ & Отсутствие & 37 \\
\hline 6 & M & 50 & 18 & Пенис & Изъязвленная бляшка & 14 & Частичный ответ & Нет данных & Нет данных \\
\hline
\end{tabular}





Наглядные примеры успешно вылеченных пациентов. Изъязвленная опухоль на левом предплечье пациента 1 до (A) и через 3 мес. после последнего сеанса лечения (В). Опухоль на левой руке пациента 4 до (C), через 2 нед. (D) и через 4 мес. (E) после последнего сеанса лечения. Бляшка на левом бедре пациента 4 до (F), через 3 нед. (G) и через 12 мес. (H) с момента последнего сеанса лечения

\section{Обсуждение}

73\% из всех обработанных очагов ГМ проявили полную клиническую ответную реакцию (ПОР). Эти данные сопоставимы с результатами предыдущих подобных исследований. P. Wolf и соавт. [8] сообщили в 1994 г. о полной клинической ответной реакции (ПОР) 3 из 3 обработанных очагов. В 2001 г. D. Edstrom и соавт. [9] сообщили о ПОР 7 из 12 обработанных очагов, а в 2008 г. эти же авторы [10] подвели итоги долгосрочного последующего наблюдения, показав, что обработанные очаги не проявляли признаков рецидива, несмотря на то, что у большинства пациентов наблюдалось дальнейшее про- грессирование заболевания в других очагах. C. Zane и соавт. [11] сообщили о ПОР 4 из 5 обработанных очагов, a S. Kim и соавт. [12] - 5 из 10 очагов. В ходе единственного перспективного контролируемого исследования, проведенного к настоящему времени, была отмечена ПОР 11 из 21 обработанного очага, а общий показатель клинической ответной реакции составил 75\% [13]. В большинстве случаев использовали МАЛ-ФДТ, тем не менее АЛК-ФДТ также оказалась эффрективной. В течение всего периода наблюдений рецидивов излеченных очагов не выявлено. Тем не менее у одного пациента появились два новых очага в непосредственной близости от ранее 
излеченных очагов. Аналогичные результаты были отмечены в ходе предыдущих исследований $[10,13]$.

В настоящее время показано лечение ГМ на ранних стадиях при помощи местной лучевой терапии, топической фототерапии, топической химиотерапии или сильнодействующих топических стероидов только при наличии одного или нескольких очагов [3]. Использование ФДТ обладает рядом преимуществ по сравнению со следующими видами лечения:

1. По сравнению с топической химиотерапией ФДТ не обладает системной токсичностью.

2. В отличие от фототерапии или лучевой терапии ФДТ не обладает канцерогенными свойствами.

3. Для ФДТ обычно нужно всего нескольких сеансов в отличие от топической фототерапии или топических стероидов, для которых необходимо проведение повторных сеансов в течение нескольких недель.
4. Косметические результаты являются хорошими.

5. Всю процедуру ФДТ в полном объеме выполняет один медицинский работник, и эта процедура подходит и пациентам, плохо соблюдающим режимы лечения.

K недостаткам ФДТ относится тот фракт, что облучение часто вызывает болевые ощущения, и процедура является относительно дорогой. Вместе с тем следует отметить, что боль можно облегчить при помощи недавно введенной в действие процедуры ФДТ дневным светом $[14,15]$. Тем не менее данные об эфрфективности ФДТ дневным светом при лечении пациентов с ГМ до сих пор отсутствуют.

В заключение отметим, что МАЛ-ФДТ является эффективным вариантом лечения солитарного ГМ или с несколькими очагами. Скорее всего, частота рецидивов является низкой. Соответственно, необходимы долгосрочные контролируемые наблюдения для проверки этих данных. I

\section{Литература}

1. Willemze R., Jaffe E.S., Burg G., Cerroni L., Berti E., Swerdlow S.H. et al. WHO-EORTC classification for cutaneous Iymphomas. Blood. 2005; 105 (10): 376885. Epub 2005/02/05.

2. Jawed S.I., Myskowski P.L., Horwitz S., Moskowitz A., Querfeld C. Primary cutaneous T-cell lymphoma (mycosis fungoides and Sezary syndrome): part I. Diagnosis: clinical and histopathologic features and new molecular and biologic markers. Journal of the American Academy of Dermatology. 2014; 70 (2): 205 e1-16; quiz 21-2. Epub 2014/01/21.

3. Jawed S.I., Myskowski P.L., Horwitz S., Moskowitz A., Querfeld C. Primary cutaneous T-cell lymphoma (mycosis fungoides and Sezary syndrome): part II. Prognosis, management, and future directions. Journal of the American Academy of Dermatology. 2014; 70 (2): 223 e1-17; quiz 40-2. Epub 2014/01/21.

4. Willemze R., Dreyling M. Primary cutaneous lymphomas: ESMO Clinical Practice Guidelines for diagnosis, treatment and follow-up. Annals of oncology: official journal of the European Society for Medical Oncology / ESM0. 2010; 21 Suppl 5: v177-80. Epub 2010/06/29.

5. Braathen L.R., Szeimies R.M., Basset-Seguin N., Bissonnette R., Foley P., Pariser D. et al. Guidelines on the use of photodynamic therapy for nonmelanoma skin cancer: an international consensus. International Society for Photodynamic Therapy in Dermatology, 2005. Journal of the American Academy of Dermatology. 2007; 56 (1): 125-43. Epub 2006/12/28.
6. Wennberg A.M. Pain, pain relief and other practical issues in photodynamic therapy. The Australasian journal of dermatology. 2005; 46 Suppl 3: S 3-4; discussion S 23-5. Epub 2005/04/30.

7. Warren C.B., Karai L.J., Vidimos A., Maytin E.V. Pain associated with aminolevulinic acid-photodynamic therapy of skin disease. Journal of the American Academy of Dermatology. 2009; 61 (6): 1033-43. Epub 2009/11/21.

8. Wolf P., Fink-Puches R., Cerroni L., Kerl H. Photodynamic therapy for mycosis fungoides after topical photosensitization with 5-aminolevulinic acid. Journal of the American Academy of Dermatology. 1994; 31 (4): 678-80. Epub 1994/10/01.

9. Edstrom D.W., Porwit A., Ros A.M. Photodynamic therapy with topical 5-aminolevulinic acid for mycosis fungoides: clinical and histological response. Acta dermato-venereologica. 2001; 81 (3): 184-8. Epub 2001/09/18.

10. Edstrom D.W., Hedblad M.A. Long-term follow-up of photodynamic therapy for mycosis fungoides. Acta dermato-venereologica. 2008; 88 (3): 288-90. Epub 2008/05/16.

11. Zane C., Venturini M., Sala R., Calzavara-Pinton P. Photodynamic therapy with methylaminolevulinate as a valuable treatment option for unilesional cutaneous T-cell Iymphoma. Photodermatology, photoimmunology \& photomedicine. 2006; 22 (5): 254-8. Epub 2006/09/05.
12. Kim S.T., Kang D.Y., Kang J.S., Baek J.W., Jeon Y.S., Suh K.S. Photodynamic therapy with methyl-aminolaevulinic acid for mycosis fungoides. Acta dermatovenereologica. 2012; 92 (3): 264-8. Epub 2011/12/16.

13. Quereux G., Brocard A., Saint-Jean M., Peuvrel L., Knol A.C., Allix R. et al. Photodynamic therapy with methyl-aminolevulinic acid for paucilesional mycosis fungoides: a prospective open study and review of the literature. Journal of the American Academy of Dermatology. 2013; 69 (6): 890-7. Epub 2013/09/18.

14. Rubel D.M., Spelman L., Murrell D.F., See J.A. Hewitt D., Foley P. et al. Daylight PDT with methyl aminolevulinate cream as a convenient, similarly effective, nearly painless alternative to conventional PDT in actinic keratosis treatment: a randomised controlled trial. The British journal of dermatology. 2014. Epub 2014/05/28.

15. Wiegell S.R., Wulf H.C., Szeimies R.M., BassetSeguin N., Bissonnette R., Gerritsen M.J. et al. Daylight photodynamic therapy for actinic keratosis: an international consensus: International Society for Photodynamic Therapy in Dermatology. Journal of the European Academy of Dermatology and Venereology: JEADV. 2012; 26 (6): 673-9. Epub 2012/01/04.

об авторах:

Р.Э. Хангер - д.м.Н., доктор философии, отделение дерматологии, Бернский университет, клиника Инзельшпиталь, Швейцария

\section{Конфликт интересов}

Авторы заявляют об отсутствии потенциального конфликта интересов, требующего раскрытия в данной статье 